\title{
La Chromobactériose animale et humaine
}

\author{
par L. JOUBERT et NGUYEN-VAN-LIEM (*)
}

\section{DÉFINITION}

La chromobactériose est une maladie infectieuse, virulente, inoculable mais peu ou pas contagieuse, commune à l'homme et à diverses espèces d'animaux domestiques et sauvages.

Exolique, d'essence hydrotellurique, non intertransmissible d'animal à animal ou à l'homme, elle est due à une bactérie spécifique, appartenant au genre Chromobacterium (Chromobacterium violaceum ou bacille violet).

D'allure généralement septicémique, peu caractéristique et souvent mortelle, la chromobactériose s'accompagne d'abcès et de tubercules viscéraux qui réclament une discrimination souvent difficile, avec la tuberculose, la morve et la mélioidose.

\section{IMPORTANCE}

L'importance économique de la chromobactériose est relativement faible en raison de son apparente rareté. Néanmoins, le caractère ubiquitaire et hydrotellurique de cette maladie tropicale force l'intérêt, d'autant que les sondages sérologiques récemment entrepris la signalent comme plus répandue qu'elle ne le paraît à première vue.

L'aspect hygiénique de la chromobactériose doit inciter le vétérinaire d'outre-mer à l'étudier plus avant, en raison de l'existence de la maladie chez l'Homme, sans qu'ait pu cependant être encore établie la preuve de l'origine animale de l'infection humaine. Aussi serait-il prématuré de considérer la chromobactériose comme une zoonose au sens propre du terme, bien que la maladie de l'homme ou de l'animal tire son origine commune du sol et des eaux.

(*) Pour les détails consulter

N Guyen-Van-Liem. - La chromobactérıse humaine et animale. Etude de quatre souches de Chromobacterium violaceum. Thèse Doct. Vétér. Lyon 1957. Ed. « Au Manuscrit 》 Alfort.

\section{HISTORIQUE}

Il est difficile de se faire une idée sur lá date d'apparition de cette maladie, car elle a été longtemps confondue avec d'autres infections telles que la pasteurellose ou la fièvre charbonneuse.

C'est Wolley (25) qui, en 1904, étudia la maladie pour la première fois, à Manille, aux îles Philippines, et fit une description originale d'une souche pathogène isolée de trois buffles morts, d'où le nom de Chromobacterium violaceum manilæ qu'il donna à la bactérie.

La même année, Breaudat (4) fit l'étude à Saïgon d'un germe saprophyte chromogène isolé de l'eau, qu'il propose de dénommer Bacillus violaceum acetonicus, en raison du dégagement d'acétone dans les milieux de culture.

En 1922, Broudin (6) rencontra un cas d'infection mortelle chez un sanglier qu'on avait 'capturé dans un puits à Tam Son Nhât, près de Saïgon.

Floch et De Lajudie (13) décrivirent en 1943, un cas septicémique de chromobactériose chez un buffle en Guyane.

Enfin en 1954, Audebaud et coll. (1) étudièrent, à l'Institut Pasteur de Brazzaville, une souche de Chromobacterium violaceum isolée d'un cas mortel chez un singe.

La même année, W. L. Sippel et coll. (19), aux Etats-Unis, relevèrent des cas mortels sur les porcs et expérimentèrent chez la même espèce par voies orale et nasale.

En ce qui concerne l'infection humaine, Sneath et coll. (20) rapportent, dans « The Lancet 》, 13 observations enregistrées de 1927 à 1953. Presque toutes concernent des cas mortels : 3 cas observés aux Etats-Unis, 10 en Malaisic.

Le cas le plus récent est rapporté et étudié par Darrasse, Mazand, Guidicelli et Camain (10) à l'Institut 
Pasteur de Dakar, qui résument remarquablement les caractères cliniques et bactériologiques de l'affection

Ajoutons qu'une étude très récente (1956 et 1957) de Leifson (15), Sneath et Eltinge (12) fait reconsidérer le problème bactériologique of taxonomique du germe.

\section{ESPÈCES AFFECTÉES}

\section{$1^{\circ}$ Dans les conditions naturelles.}

La chromobactériose atteint l'homme, le singe, le buffle, le bceuf, le sanglier et le porc.

La maladie spontanée a été observée à l'état sporadique chez le buffle et chez le porc, mais on n'a relevé jusqu'à próscnt aucun cas de maladie naturelle chez le cheval, le chien et les petits rongeurs.

\section{$2^{\circ}$ Dans les conditions expérimentales.}

La maladie est aisément transmissible aux petits animaux de laboratoire: souris, cobaye et lapin. 'Ces petits rongeurs s'avèrent sensibles par toutes les voies: injection sous-cutanée, intrapéritonéale, intraveineuse, intranasale. Mais ils résistent quand on les nourrit avec des aliments aspergés avec du bouillors de culture.

D'autre part, on ne note aucun cas de transmission de la maladie par contact de l'homme ou des animaux avec les animaux malades.

\section{RÉPARTITION GÉOGRAPHIQUE}

C'est surtout dans les pays tropicaux et subtropicaux que sévit la chromobactériose. L'humidite du sol, associée avec la chaleur, favorise les grandes poussées de la maladie, qui coïncident d'ordinaire avec la saison des pluies. La nature du terrain peut être considérée comme un facteur adjuvant prépondérant dans l'étiologie de l'infection. En Malaisic comme au Vietnam, les terres alluvionnaires, à sous-sol imperméable formant de nombreux marécages, sont le plus souvent des terres favorables au dévaloppement de la maladie. Notons aussi que la plupart des animaux naturellement sensibles marquent une dilection certaine pour les eaux fangeuses.

Des souches pathogènes pour l'animal ont été isolées dans différents Instituts Pasteur d'outre-mer: Indochine, Guyane, Afrique Equatoriale et Occidentale.

La maladie a été décelée dans les îles Philippines, en Asie (Indochine surtout, et en Malaisie), en Afrique tropicale et en Amérique (Guyane, régions chaudes et humides des Etats-Unis).
Nous n'avons pas connaissance que cette maladie ait été reconnue dans les pays à climat tempéré et froid, ce qui nous permet de penser que la chromobactériose reste l'apanage des pays tropicaux et subtropicaux dont le climat comporte des alternatives de chaleur et d'humidité.

\section{EPIDEMIOLOGIE}

Chromobacterium violaceum est très répandu dans la nature: il se rencontre très fréquemment sur le sol, dans les eaux, associé au colibacille $(24,2,7)$. Pour certains auteurs, la présence des bacilles violets traduirait, à elle seule, une souillure fécale, opinion réfutée par d'autres auteurs.

Brisou (5) le rencontre aussi dans l'eau de mer : ce germo cst douć do proprićtés chitinolytiques grâce à la présence d'une enzyme, la chitinase, qui transforme la chitine en chitosan, ce qui lui permet de se développer dans le milieu marin en s'attaquant aux dépôts de chitine si abondants parfois dans la mer.

Au Vietnam, on rencontre souvent Chromobacterium violaceum à l'état saprophyte dans les eaux non chlorurées et, au cours de la numération des germes totaux, en culture sur gélose, dans les Services de Contrôle des Eaux, on isole le bacille violet assez fréquemment.

Comme les germes abondent surtout dans les eaux fangeuses, lid chromobactériose affecte souvent les buffles et les porcs, animaux qui se délectent dans la boue et dans les marécages.

Ainsi, les conditions locales, telluriques et atmosphériques semblent ici réunies pour créer des foyers intenses de maladie. Cependant, on no remarque pas de foyers de chromobactériose à marche envahissante: la maladie, hydrotellurique, reste essentiellement sporadique ou enzootique à faible diffusion, Il s'agit même vraisemblablement de pseudo-enzooties, illustrant le rôle prépondérant du terrain, du mode d'élevage et de l'alimentation: la maladie naitrait alors sous l'influence de conditions similaires chez plusieurs animaux à la fois, à partir d'une source de virus tellurique commune.

\section{BACTÉRIOLOGIE}

I - NOMENCLATURE ET SYSTEMATIQUE. Deux problèmes restent difficiles à résoudre pour nommer et classer les bactéries responsables de la chromobactériose en raison:

- d'une part, de l'incertitude entachant la dénomination même de l'espèce bactérienne,

- d'autre part, de la fragilité des critères de classification au sein du genre Chromobacterium, 
l'une et l'autre dues au petit nombre des publications sur ce sujet et aussi à la description souvent incomplète donnée par les auteurs des sonches qu'ils ont isolées.

La chromobactériose admet classiquement comme responsable Chromobacterium violaceum, très proche de 2 autres variétés: Chr. manilæe et Chr. laurentium, toutes trois secrétant un pigment violet soluble dans l'éthanol et insoluble dans le chloroforme.

Les differences physiologiques séparant ces 3 espèces ont été étudiées avec précision par Leifson (15) et Eltinge (12) et l'accord ne s'est pas réalisé sur le nombre d'espèces authentiques contenues dans le genre Chromobacterium, ni sur los critèros déterminatifs (culturc à $37^{\circ}$, fermentation et oxydation des hydrates de carbone, attaque des nitrates etc.). Par ailleurs, l'étude spectrophotométrique des pigments bactériens a permis à Gilman (14) de diviser les germes de ce genre en 3 variétés: Chr. violaceum, Chr. amethystinum, Chr. janthinum.

On voit la nécessité d'une étude technique et taxonomique plus approfondie, pour disposer désormais d'une classification claire et sûre.

Seule, la situation du genre Chromobacterium est précise parmi les espèces bactériennes. Il appartient à l'ordre des Eubacteriales, famille des Pseudomonadaceæ, tribu des Chromobactereo. Cette famille, extrêmement vaste, comprend 3 tribus avec 13 genres de bactéries, se présentant toutes sous forme de bâtonnets allongés, droits ou incurvés, asporulés, ne prenant pas le Gram, mobiles ou immobiles, aquatiques ou terrestres, la plupart pathogènes pour les plantes et quelques-unes pour les animaux et même pour l'homme.

Selon la révision faite en 1948 par J. Magrou et A. R. Prevost (16), la tribu III des Chromobacterece compte 4 genres:

10 Chromobacterium (Bergonzini).

$2^{\circ}$ Flavobacterium (Bergey).

3o Protaminobacter (den Dooren de Jong).

$4^{\circ}$ Serratia (Bizio).

\section{II - LE GERME}

La description qui suit est partie la compilation des descriptions classiques du germe (en particulier par Topley et Wilson (23) et partie le fruit de nos propres observations.

Morphologie. - C'est un bâtonnet droit de 1,5 $\mu$ 5 à 3 . de long sur $0,6 \%$ de large, à extrémités arrondies, ne restant pas coloré par la méthode de Gram, acapsulé, asporulé et mobile. Cette mobilité faible (culbute sur place) est due à la présence d'un flagelle qui n'a pas échappé à l'observation de différents auteurs. Sippel et Coll. (19), après l'examen au microscope électronique, signalent l'existence d'un flagelle simple monotriche implanté à l'une des extrémités de la bactérie. Ce cil polaire. est unique ( $\mu=2,19 \mu$ ) ou s'aćcompagne d'un ou plusieurs cils latéraux à p plus faible $(1,30$ y.environ).

La coloration du cytoplasme est uniforme et les germes apparaissent souvent en palissades (cf. Corynebacteries) ou figurent un $V$ (formes de division). Les formes filamenteuses sont inconnues.

Culture - Chromobacterium violaceum cultive facilement sur tous les milieux usuels, en aérobiose de préférence, à la température de $37^{\circ}$. (*) Son $\mathrm{pH}$ optimum est situé entre 7 et 8 , les $\mathrm{pH}$ extrêmes varient entre 5 et 10 .

Dans le bouillon ordinaire ou dans l'eau peptonée, la culture se traduit par un trouble uniforme, un anneau violet apparaissant en quelques jours à la surface. Sur les milieux solides (gélose ordinaire, sérum coagulé), les colonies sont violettes, arrondies, bombées, humides, à bords nets. Ia meilleure croissance est obtenue en présence de l'amidon de riz (9)

Cependant la propriété la plus caractéristique dans les cultures est la production des pigments.

\section{Biochimie.}

Pouvoir protéolytique. La gélatine ensemencée en piqûre centrale ou en masse, est liquéfiée en 24 à 48 heures surtout en surface, où le développement microbien est maximum, pour s'annuler en culot. Le sérum coagulé est rapidement digéré et des cupules de lyse se creusent dès la $24^{\mathrm{e}}$ ou la 48 e heure.

Le lait tournesolé est réduit léntement en 2 à 8 jours, mais n'est pas coagulé.

L'hémolyse des globules rouges de lapin est faible et inconstante.

\section{Pouroir saccharolytique.}

Il est variable avec les souches. En général le glucose, le maltose, le lévulose, le saccharose et la salicine sont utilisés avec ou sans gaz.

\section{Produits formés dans les milieux de culture.}

1. Pigment. Le pigment violet, ou "violacéine " a été l'objet d'études approfondies de Satory et coll. (17) et de Strong (21). Il appartient au groupe chimique des anthocyanines. Il reste attaché étroitement au corps bactérien et ne diffuse pas, par

(*) Selon Leifson, Chr. tiolaceum typique ne cultiverait pas à $37^{\circ}$. 
conséquent, dans le milieu de culture liquide ou solide, alors que la masse bactérienne est entièrement teintée en violet.

L'extraction du pigment s'effectue par filtration sur Seitz d'une culture sur gélose laissée à la température du laboratoire pendant 4 à 5 jours. Le produit est émulsionné dans l'acétone, qui dissout le pigment. A l'encontre de la pyocyanine du genre Pseudomonas, le pigment violet est peu soluble dans l'éther, insoluble dans l'eau, le chloroforme, le toluene et le benzène; il est soluble en revanche dans l'alcool et l'acétone.

Rẻduite en un leucodérivé par des réducteurs tels que le thiosulfate de sodium, la solution acétonique du pigment prend une teinte jaune pâle qui, au contact du bichromate de potassium, s'oxyde et reprend la couleur violette.

Les travaux de Satory (17), de Gilman (14), de Audebaud (1) et de Darasse (10) insistent sur la courbe spectro-photométrique de la solution acétonique du pigment et la désignent comme un excellent critère pour séparer les différentes bactéries chromogènes les unes des autres. On y note la concordance du résultat suivant : le maximum d'absorption se trouve à 580 millimicrons et le minimum à 440 millimicrons.

La formation du pigment est favorisée, par la lumière et l'oxygène, à la surface de certains milieux solides tels que la pomme de terre ou l'amidon de haricot. Il se développe mal en bouillon ou dans la profondeur des cultures.

2. Indol. Pas de production.

3. Hydrogène sulfuré. Aucun dégagement.

4. Hydrolyse de l'urée. Absente.

5. Acétylméthyl carbinol. Non révélable.

6 . Utilisation du citrate de sodium. Plus ou moins rapide (24 à 48 h.) en milieu solide de Simmons.

7. Réduction des nitrates en nitrites. Constante.

8. Test au rouge de méthyle : Positif.

Chimio et antibiosensibilité. Par la méthode des diffusions en gélose, la scnsibilité aux sulfamides signale le septoplix et le thiazomide, celle aux antibiotiques, la streptomycine et le chloramphénicol comme les plus actifs.

\section{III. - LA TOXINE}

10 Exotoxine. - Préparée en milieu liquide en $24 \mathrm{~h}$, centrifugée et filtrée sur bougie L3, l'exotoxine a été étudiée à partir de 4 souches.

a) Propriétés hémolytiques in vitro. - Dans une série de tubes, la toxine est diluée progressivement jusqu'au 1/20,000. L'épreuve d'hémolyse par contact avec une suspension de globules rouges de lapin à 1 pi 100 , après une heure à $37^{\circ}$ puis une houre à la température du laboratoire n'a révélé aucun pouvoir hémolytique.

b) Propriètés dermo-nécrosantes. - Le même filtrat provoque chez le lapin, par voie intradermique, à la dose de $1 / 10$ de $\mathrm{cm}^{3}$ après $24 \mathrm{~h}$. une escarre suintante superficielle peu étendue qui cicatrise facilement et rapidement.

c) Propriété létale. - L'inoculation de $2 \mathrm{~cm}^{3} \mathrm{de}$ filtrat de stérilité reconnue, à un cobaye, par voie sous-cutanée, n'a produit aucune réaction. En revanche, l'action du filtrat semble beaucoup plus active chez le lapin, en injection intraveineuse : $2 \mathrm{~cm}^{3}$ de ce produit inoculé dans la veine marginale de l'oreille tuent l'animal au bout de $6 \mathrm{~h}$., alors qu'un lapin témoin ayant reçu la même quantité du bouillon de base stérile, ne présente aucun symptôme particulier.

L'animal présente de l'hyperthermie, une prostration extrême et meurt dans un véritable état de choc. A l'autopsie, sont notées les lésions suivantes : congestion importante des poumons et du foie, lésions hémorragiques de l'estomac. La culture du sang du coeur, de la moelle et des organes reste négative.

En dépit de ces premiers résultats, il apparaît néanmoins difficile d'assimiler ce poison à une véritable toxine soluble du germe en raison de sa nocivité relative. Une étude plus approfondie serait nécessaire pour pouvoir apprécier la toxicité de cette bactérie, niée par Darrasse (10).

Hémolysine. En milieu solide au sang de lapin, certaines souches produisent une hémolyse partielle des hématies, semblable à l'hémolyse 5 du staphylocoque (halo d'hémolyse péribactérien, sans clarification totale du milieu).

$2^{\circ}$ Endotoxine - Selon les données de Sneath (20), le bacille ne semble pas héberger un constituant endotoxique du type Boivin-Topley-Morgan. Confirmant ce travail, nous n'avons pu révéler de fraction nettement toxique après traitement des germes par l'acide trichloracétique $\mathrm{N} / 2$.

\section{IV. - BIOLOGIE.}

Pouvoir pathogène - Les diverses souches de Chr. violaceum montrent un pouvoir pathogène extrêmement nuancé et le problème des variations quantitatives de la virulence des souches fraîchement isolées doit se placer parmi les préoccupations majcurcs du bactériologiste.

Au chapitre du pouvoir pathogène qualitatif. le pantropisme du germe rend compte de la grande fréquence des Septicémies chromo-bactériennes et des lésions ganglionnaires et réticuloendothéliales qu'engendre cette espèce. Il s'associe à un pneumo- 
TABLEAU I

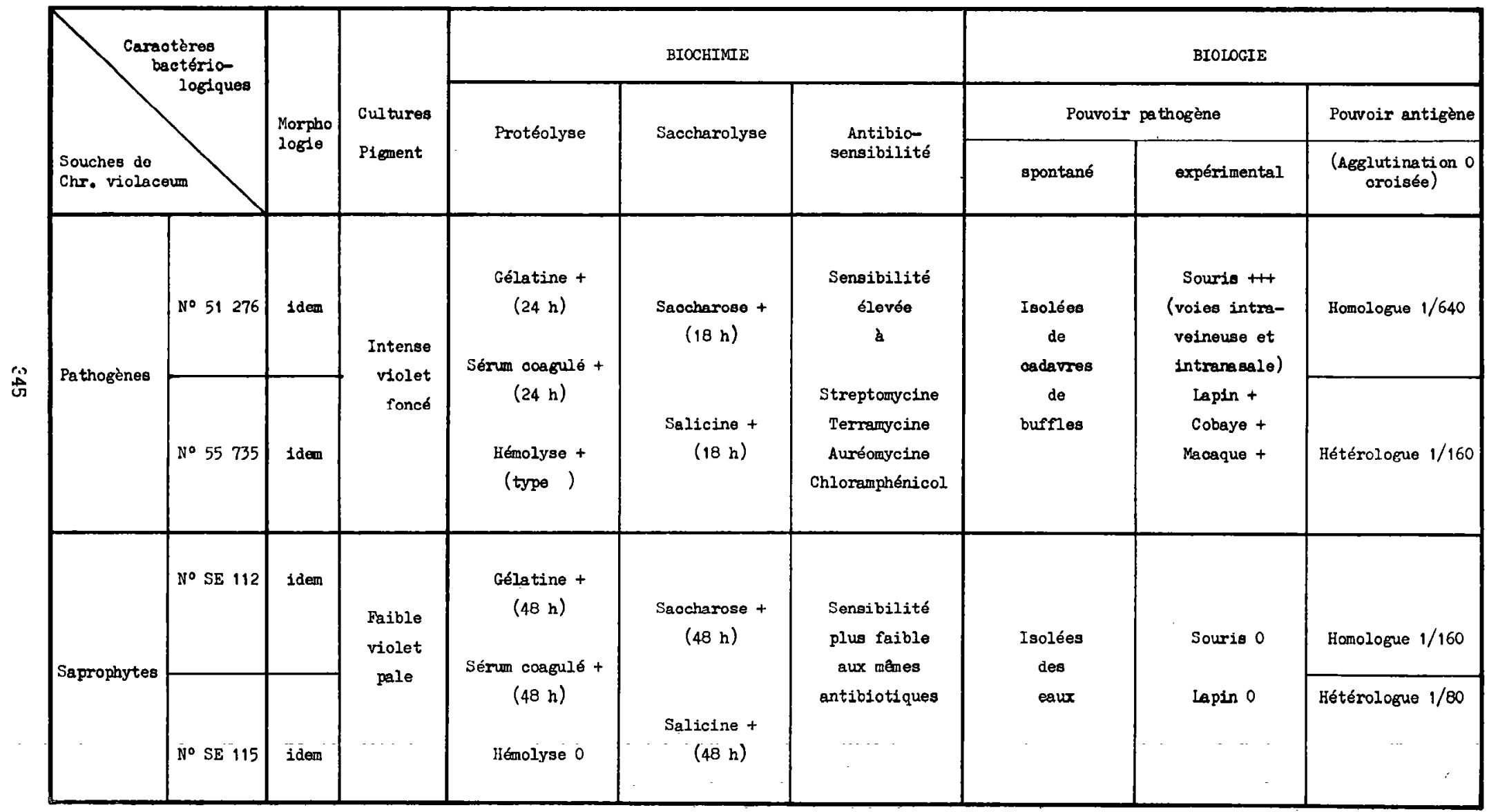


tropisme révélé dans l'infection spontanée par la fréquence des lésions pulmonaires et, à l'expérimentation, par la réussite régulière des inoculations intranasales, chez la souris notamment.

La chromobactériose est iacile à reproduire par inoculation aux animaux de laboratoire.

Dans une expérience, Audebaud et coll. (1) ont provoqué la maladie sur un Cercopithecus cephus par injection sous-cutanée d'une émulsion de culture de $24 \mathrm{~h}$. du germe; ils constatent que l'inoculation provoque; an 5 e jour, la mort du singe, qui présente des lésions identiques à celles observées chez le premier animal atteint.

Darrasse et coll. (10) ont inoculé des germes vivants à la souris et au cobaye. La souris meurt en quelques heures à un jour, le cobaye, en 2 à 3 jours.

A l'autopsie, on trouve des abcès hépatiques avec des foyers de nécrose qui renferment des bactéries inoculées.

Schattenberg (18) a réussi à provoquer la maladie chez le lapin avec terminaison fatale, en introduisant dans la patte une écharde de bois préalablement plongée dans unc culture de Chromobactcrium violaceum.

Pour obéir aux lois naturelles, Sippel et coll. (19) ont expérimenté l'infection chez des porcs par voie nasale et orale. Leur expérience démontre qu'un porc de poids moyen peut être tué en 2 à $\theta$ jours par $0,2 \mathrm{~cm}^{3}$ d'une culture de Chromobacterium violaceum en bouillon de 24 heures introduite par voie nasale.

Pour ces auteurs, la maladie naturelle semble résulter des infections par voies orale et nasale.

La souris nous paraît l'animal réactif de choix par voies inilranasale el intraveineuse.

Pouvoir antigène, immunigène et allergène. Ce chapitre, pou ćtudić, se crcusc de nombrcuscs lacunes. L'immunité dans les chromobactérioses est inconnue, en raison de la rareté de l'infection spontanée, qui n'a pas posé de pressants problèmes de vaccination. Par ailleurs, le pouvoir allergique n'a pas été approfondi.

Seule l'antigénicité somatique $(0)$ et flagellaire $(H)$ de la bactérie a été étudiée par Sneath (20) sans qu'aient été précisées les relations entre toxines, pouvoirs pathogène et antigène chez des souches saprophytes isolées des eaux et une souche pathogène issue d'une infection urinaire de l'Homme.

5. VARIABILITÉ de Chr. Violaceum et RELATIONS ENTRE LES CARACTÉRES MORPHOLOGIQUES,

\section{CULTURAUX, BIOCHIMIQUES ET BIOLOGIQUES DE LA BACTÉRIE.}

L'éventail extraordinairement étalé de la virulence des souches de Chr. Violaceum les unes vis-à-vis des autres en fonction de l'origine de leur isolement, incitait à étudier comparativement 2 groupes de souches, l'un formé de bactéries pathogènes, isolées de chromo-bactérioses bubalines, l'autre de bactéries saprophytes, isolées des eaux. (*)

Quatre souches de l'Institut Pasteur de Saïgon, 2 pathogènes 51.276 et 55.735 isolées de buffles morts 2 saprophytes S E 112 et S E 115 isolées des eaux, ont été expertisées comparativement sur les chapitres de la morphologie, des cultures, de la biochimie et de la biologie (virulence et pouvoir antigène) dans le but de déceler chez cette espèce les caractéristiques du pouvoir pathogène in vivo sur l'animal (test de certitude) et in vitro (tests de présomption).

De nombreux caractères s'avèrent communs et seuls seront retenus ceux qui séparent les souches pathogènes des souches saprophytes.

La lecture du tableau I renseigne sur les chapitres suivants :

10 Morphologie - Elle ne permet en aucun cas de discerner les 2 groupes de bactéries.

$2^{\circ}$ Culture. - Le pigment violet colorant la masse bactérienne est plus intense, de teinte plus foncée pour les souches pathogènes.

\section{$3^{\circ}$ Biochimie.}

- La protéolyse (liquéfaction de la gélatine, attaque du sćrum coagulć ct hómolyse des hématios de lapin en milieu géloś́) est plus nette et plus rapide pour les souches pathogènes.

- La saccharolyse (fermentation du saccharose et de la salicine) est plus précoce chez les bactéries virulentes.

- L'antibiosensibilité est plus élevée pour les souches pathogenes vis-à-vis de la streptomycine, de la terramycine, de l'auréomycine et du chloramphénicol que pour les souches saprophytes. Le détail de ce test (milieu gélosé et méthode des disques) est donné par le tableau II :

(*) Nos remerciements vont aux Docteurs Marneffe, Fournier, Destournes, Nguyen Ba Luong et Chambon, de l'Institut Pasteur à Sajgon, à qui reviennent, pour des motifs divers, une très grande part de ce travail. 
TABLEAU II

\begin{tabular}{|c|c|c|c|c|c|c|c|c|}
\hline \multirow{3}{*}{ Antibiotiques } & \multicolumn{4}{|c|}{ SOUCHES PATHOGENES } & \multicolumn{4}{|c|}{ SOUCHES SAPROPHYTES } \\
\hline & \multicolumn{2}{|r|}{$N^{\circ} 51.276$} & \multicolumn{2}{|r|}{$N^{\circ} 55.735$} & \multicolumn{2}{|r|}{$N^{\circ} \mathrm{SE} .112$} & \multicolumn{2}{|r|}{$N^{\circ} \mathrm{SE} .115$} \\
\hline & $\mathrm{Dm} / \mathrm{m}$ & Sensibilité & $\mathrm{Dr} / \mathrm{m}$ & Sersibilité & $\mathrm{Dra} / \mathrm{m}$ & Sensibilịté & $\mathrm{Dm} / \mathrm{m}$ & Sensibilité \\
\hline Pénicilline & $0-32$ & $\mathrm{~S}>5 \mathrm{JO} / \mathrm{cm}^{3}$ & $0-32$ & S) $5>00 / \cos ^{3}$ & $0-32$ & $\mathrm{~S}>5 \mathrm{vO} / \mathrm{cm}^{3}$ & $0-32$ & $s>500 / \mathrm{cm}^{3}$ \\
\hline Auréomycine & $14-17$ & $\mathrm{~S}=1 \mu \mathrm{g} / \mathrm{cm}^{3}$ & $12-17$ & $\mathrm{~S}=1 \mathrm{\mu E}^{\mathrm{s}} / \mathrm{cs}^{3}$ & $26-17$ & $\mathrm{~s}<0,5 \mu \mathrm{g} / \mathrm{cm}^{3}$ & $25-17$ & $\mathrm{~s}<0,5 \mu \mathrm{g} / \mathrm{cm}^{3}$ \\
\hline Chloramphénicol & $32-27$ & $1<S<2 \mu \mathrm{g} / \mathrm{cm}^{3}$ & $30-27$ & $S=2 \mu \mathrm{g} / \mathrm{cm}^{3}$ & $40-27$ & $\mathrm{~s}<0,5 \mu \mathrm{g} / \mathrm{cm}^{3}$ & $38-25$ & $\mathrm{~s}<0,5 \mu \mathrm{g} / \mathrm{cm}^{3}$ \\
\hline Terramycine & $16-20$ & $S=1 \mu \mathrm{E} / \mathrm{cm}^{3}$ & $16-20$ & $\mathrm{~S}=1 \mathrm{\mu g} / \mathrm{cm}^{3}$ & $32-20$ & $\mathrm{~S}<0,5 \mu \mathrm{g} / \mathrm{cm}^{3}$ & $33-21$ & $\mathrm{~S}<0,5 \mu \mathrm{g} / \mathrm{cm}^{3}$ \\
\hline Streptomycine & $30-28$ & $0,5<\mathrm{s}<1 \mu \mathrm{g} / \mathrm{cm}^{3}$ & $22-28$ & $S=1 \mu g / \mathrm{cm}^{3}$ & $32-28$ & $\mathrm{~s}<0,5 \mu \mathrm{g} / \mathrm{cm}^{3}$ & $34-29$ & $\mathrm{~s}<0,5 \mu \mathrm{g} / \mathrm{cm}^{3}$ \\
\hline Bacitracine & $0-24$ & $\mathrm{~S}) 100 \mathrm{U} / \mathrm{cm}^{3}$ & $0-24$ & $\mathrm{~s}>100 \mathrm{v} / \mathrm{cm}^{3}$ & $0-24$ & $\mathrm{~S}>100 \mathrm{U} / \mathrm{cm}^{3}$ & $0-24$ & $\mathrm{~S}>100 \mathrm{v} / \mathrm{cm}^{3}$ \\
\hline Tétraoyoline & $7-20$ & $5<5<10 / \mathrm{cm}^{3}$ & $8-28$ & $\mathrm{~S}=5 \mu \mathrm{g} / \mathrm{cm}^{3}$ & $32-20$ & $\mathrm{~s}<0,05 \mu \mathrm{g} / \mathrm{cm}^{3}$ & $31-20$ & $\mathrm{~s}<0,05 \mu \mathrm{g} / \mathrm{cm}^{3}$ \\
\hline Erythromycine & $18-28$ & $S=1 \mu g / \mathrm{cm}^{3}$ & $17-28$ & $\mathrm{~S}=3 \mu \mathrm{g} / \mathrm{cm}^{3}$ & $22-28$ & $\mathrm{~s}<0,05 \mu \mathrm{g} / \mathrm{cm}^{3}$ & $22-28$ & $\mathrm{~S}<0,05 \mathrm{\mu E} / \mathrm{cm}^{3}$ \\
\hline
\end{tabular}

\section{Biologie.}

Le pouvoir pathogène expérimental des souches isolées du buffle et considérées comme pathogènes, a été facile à mettre en évidence sur souris, cobaye, lapin et macaque alors que les souches jsolées des eaux et considérées comme saprophytes se sont révélées impuissantes à déclencher une infection expérimentale.

Le détail de cette expérimentation peut être ainsi résumé :

\section{I. - Souches pathogènes.}

I. - Inoculation sous-cutanée.

Singe. Macacus cynomolgus est sensible à l'inje ctionde Chromobacterium violaceum $\left(0,5 \mathrm{~cm}^{3}\right.$ de culture en bouillon de 24 heures sous la peau, à la face interne de la cuisse).

Quatre heures après l'inoculation apparait une hyperthermie: la température monte à $39^{\circ} 2$, puis descend le lendemain à $38^{\circ}$; vers le $3^{e}$ et le $4^{\mathrm{e}}$ jour. elle oscille légèrement au-dessus de la normale, mais remonte à nouveau vers le $5^{\mathrm{e}}$ jour $\left(39^{\circ} 8\right)$ et les jours qui suivent.

L'appétit diminue, l'animal reste couché et c'est huit jours après l'inoculation que l'état général devient le plus déficient. L'animal meurt très amaigri en hypothermie le $9 \mathrm{e}$ jour.

L'autopsie révèle l'existence de nombreux petits abcès dans le fois, le volume de l'organe restant normal. il en est de même pour la rate, mais les abcès y sont plus rares. Les poumons emphysémateux achèrent à la paroi thoracique. L'estomac est vide avec des lésions congestives et hémorragiques; les reins et les capsules surrénales sont fortement congestionnés.

L'examen histo-pathologique de ces différents organes montre qu'il s'agit d'une hépatite dégénérative of nécrosante sans stéatose, à localisation centro-lobulaire prédominante, la nécrose se faisant sur place, sans abcédation vraie, mais le squelette collagène du lobule est conservé.

Au niveau de l'estomac, existe une importante nécrose de la muqueuse s'étendant localement à la sous-muqueuse avec nécrose des parois vasculaires dans ces zones.

Dans les reins, on décèle une néphrite épithéliale pure, sans lésion glomérulaire, avec nombreux cylindres dans les collecteurs. Le cortex des surrénales montre des lésions congestives et hémorragiques.

Quant aux poumons, on note des lésions d'emphysème généralisé. Au total, l'animal a succombé à une hépato-néphrite avec congestion importante et même, parfois, lésions hémorragiques de tous les autres organes.

Des cultures à partir des lésions du foie redonnent Chromobacterium violaceum, mais l'hémo. culture du sang du cœur et l'ensemencement de la moëlle restent négatifs. 
Lapin. - Le lapin se révèle réceptif, mais avec une sensibilité qui varie avec les sujets.

Trois lapins à peu près du même poids $(1,500 \mathrm{~kg})$ ont été inoculés avec $0,5 \mathrm{~cm}^{3}$ de culture de la souche 55.735 en bouillon de 24 heures par voie souscutanée, à la face interne de la cuisse. Très rapidement, un abcès se forme au point d'inoculation. L'un de ces animaux meurt 5 jours après l'inoculation, le $2^{\mathrm{e}}$ animal est mort le $8^{\mathrm{e}}$ jour, le $3^{\mathrm{e}}$ a survécu et a été sacrifié un mois après l'inoculation.

Après sacrifice de l'animal guéri, aucune lésion suspecte ne fut relevée, la culture du sang du cœur et des différents organes reste négative.

Sur les animaux morts, l'autopsie a révélé les lésions suivantes:

Foie: congestion des vaisseaux avec micronodules nécrotiques;

Poumons : congestion très importante mais absence de nodules;

Rate: hémorragies discrètes mais le volume reste normal;

Estomac: nécrose épithéliale avec atrophie musculaire.

La culture des organes donne Chromobacterium violaceum mais l'hémoculture du coeur reste négative.

Cobaye. - Le cobaye, comme le lapin, est sensible mais avec moins de régularité que la souris.

Un lot de 5 cobayes est inoculé aver $0,5 \mathrm{~cm}^{3}$ d'une émulsion de culture de la souche 55.735 de 24 heures par voie sous-cutanée. Les animaux réagissent par une élevation thermique $\left(42^{\circ} 2\right)$, la fièvre se maintient au-dessus de la normale pendant 4 jours, au cours desquels se forme un abcès au point d'inoculation. L'abcès peut évoluer vers l'ouverture spontané, et l'animal survit alors. Sacrifié 2 mois après l'inoculation, en excellente santé l'animal montre à l'autopsie, des organes normaux et les cultures des organes et du sang du cceur restent négatives.

Pour les 4 autres animaux, l'abcès limité au point d'inoculation se résorbe vers le $6^{\mathbf{e}}$ ou $8^{\mathrm{e}}$ jour. Les animaux maigrissent puis meurent en des délais échelonnés: 2 en 10 jours, le $3^{\mathrm{e}}$ vers le $12^{\mathrm{e}}$ jour et le $4 \%, 15$ jours plus tard. A l'autopsie, les lésions relevées sont les suivantes: Ies reins sont fortement congestionnés, les surrénales se chargent, dans toutes leurs couches, de cellules éosinophiles; les poumons sont congestionnés, Par contre, dans le foie, se décèle une réticulose micronodulaire avec atteinte des cellules hépatiques. La rate présente une congestion discrète mais pas de foyers nécrotiques.

Au point inoculé, on ne relève aucune trace de l'abcès. Mais les ensemencements à partir du foie mettent en évidence Chromobacterium violaceum, tandis que la culture du sang du cœur et de la moelle reste stérile.

Souris blanche. - La souris est très sensible à l'inoculation. Dans cette série d'expériences, 6 souris reçurent par voie sous-cutanée $0,2 \mathrm{~cm}^{3}$ d'émulsion de culture de 24 heures: tous les animaux de cette série sont morts 12 à 24 heures après l'inoculation. Les lésions sont celles d'une congestion intense de tous les organes.

L'hémoculture est positive à l'autopsie et permet de retrouver Chromobacterium violaceum en culture pure.

\section{II. - Voie intraveineuse.}

Lapin. - L'injection par voie veineuse au lapin s'avère fatale pour l'animal. Après l'injection dans la veine marginale de l'oreille de $0,5 \mathrm{~cm}^{3} \mathrm{~d}^{\prime} u n e$ émulsion de 24 heures de la souche 55.735, l'animal réagit par une forte fièvre (4103), accompagnée de troubles graves: anorexie complète, respiration dyspnéique et mort 10 heures après l'inoculation.

A l'autopsie, on décèle dans les reins une congestion importante des glomérules et des capillaires interstiliels. La tate présente une extrême congestion des sinus veineux, une raréfaction lymphoïde des corpuscules de Malpighi avec macrophagie au niveau des centres germinatifs et le foie, une congestion modérée des capillaires et une absence de pigment dans les cellules de Kupfer. Une pneumonie hémorragique très accentuée apparaît à l'ouverture du thorax, mais les lésions nodulaires et nécrosantes font complètement défaut.

Dans l'ensemble, les lésions congestives et hémorragiques sont accentuées au maximum dans la rate. l'estomac et les poumons; elles sont moins marquées au niveau du foie et des reins.

L'ensemencement du sang du coeur et de la moelle fournit une culture pure de Chromobacterium violaceum.

III. - Inoculation intrapéritonéale.

Souris blanche. - Les 4 souris inoculées avec $0,2 \mathrm{~cm}^{3}$ d'une culture en bouillon de 24 heures de la même souche, par voie intra-péritonéale, meureni 8 à 10 heures après l'inoculation. A l'autopsie, sont constatées des lésions congestives extrêmement accentuées de tous les organes: foie, rate, poumons et glomérules du rein en particulier, accompagnées d'une péritonite, avec épancherment de sérosité au point inoculé.

L'hémoculture du sang et l'examen bactériologique de la moelle sont positifs.

Cobaye. - Le cobaye est plus sensible par voie intrapéritonéale que par voie sous-cutanée. 
Dans cette serie d'expériences, 5 cobayes sont inoculés avec $0,5 \mathrm{~cm}^{3}$ d'une émulsion de culture de 24 heures de la même souche, en peritoine: trois animaux meurent en 10 heures, les deux autres, après 12 heures.

Les lésions sont celles d'une septicémie: congestion intense du foie, des reins, de la rate, de la muqueuse stomacale; suffusion sanguine du péritoine avec exsudat séreux; lésions de pneumonie hémorragique, congestion intense des capsules surrénales.

La culture du sang du cceur et de la moelle est positive.

\section{IV. - Voie nasale.}

Nous avons évoqué plus haut l'expérience effectuée par L. Sippel (19) et collaborateurs: en injectant une émulsion de germe aux porcs par voie nasale on démontre l'extrême facilité de l'infection par cette voie (la dose de $0,8 \mathrm{~cm}^{3}$ d'émulsion de culture de 24 heures en instillation nasale tue un porc en 44 heures par pneumonie congestive).

Une semblable expérience a été répétée sur la série des petits animaux de laboratoire de la façon suivante: avec un compte-goutte, une émulsion de culture de 24 heures de la souche 55.735 a été instillée dans la cavité nasale de chaque animal, à raison de 2 gouttes pour la souris, 4 gouttes pour le cobayo ot 6 gouttcs pour le lapin. Voici les résultats obtenus : comme au cours des septico-pyoémies, les lésions des poumons restent constantes : pneumonie congestive avec œdème du tissu alvéolaire dans le premier cas; nodules nécrotiques plus ou moins accentués dans le second, ce qui permet de conclure que les poumons constituent un organe de prédilection pour la multiplication de la bactérie, douée, comme nous le soulignons plus haut, d'un double tropisme: partropisme et pneumotropisme.

\section{II. - Souche saprophyte.}

Pour comparer le pouvoir pathogène d'une souche virulente avec celui d'une souche saprophyte, la souche isolée des eaux SE 112 a été inoculée dans les mêmes conditions aux différents animaux de laboratoire, mais cette expérimentation s'est soldée par l'échec presque constant des' inoculations.

Cobaye. - Dans la première série d'expériences par voie sous-cutanée la dose de $0,5 \mathrm{~cm}^{3}$ d'une émulsion de culture de 24 heures de la souche.SR. "112 a été inoculée à 5 cobayes. Voici les résultats relevés : la réaction des inoculés est nettement moins forte, on note une légère hyperthermie et un abcès peu volumineux qui ne suppure pas puis se résorbe. Tous les animaux conservent un bon état général, et 2 mois après ils sont toujours en excellente santé. A l'autopsie de deux d'entre eux, aucune lésion n'est relevée et les examens d'organes, de sang et de moelle restent négatifs.

TABLEAU III

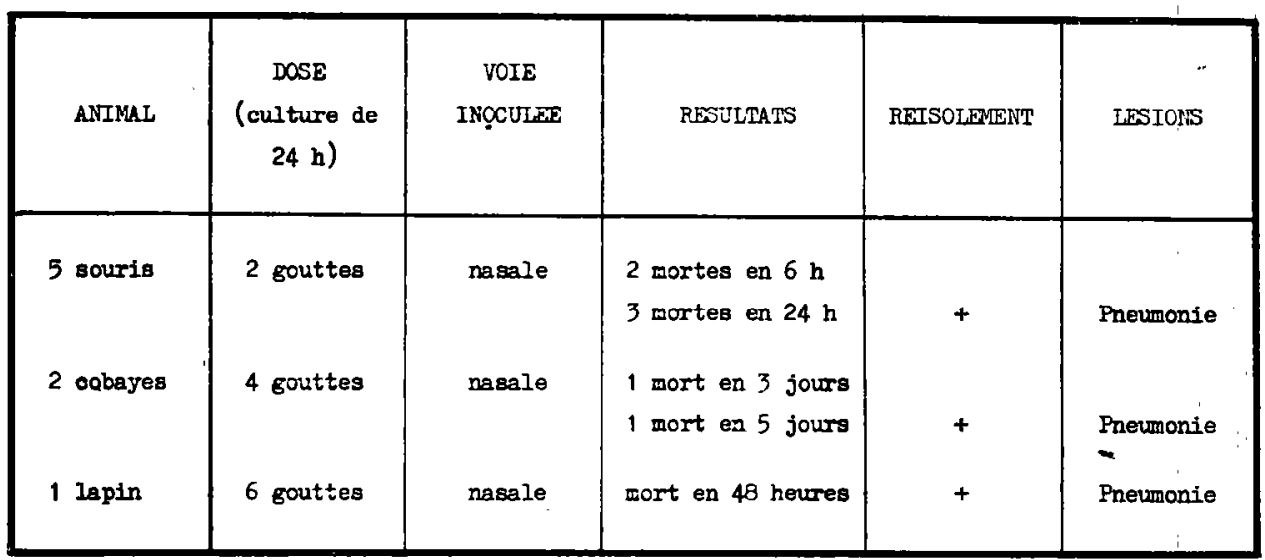

Pour les animaux réceptifs, l'introduction par voie nasale de Chromobacterium violaceum s avère donc particulièrement sévère, ce qui concorde avec les résultats obtenus par Sippel. Par ailleurs, quelle que soit la voie d'inoculation utilisée, les localisations pulmonaires prédominent dans tous les tableaux cliniques. En cas de septicemie rapidement évolutive
En péritoine, une injection de $0,5 \mathrm{~cm}^{3}$ d'une même émulsion de culture sur 5 autres cobayes se heurte à la résistance de tous les animaux. On note un amaigrissement léger pendant la premiere semaine après l'inoculation, puis l'état général s'améliore. La guerison est complète au bout de 2 semaines. 
Souris. - L'injection dans le péritoine de 4 souris de $0,5 \mathrm{mc}^{3}$ d'émulsion de la souche SE. 112 fournit les résultats suivants: deux animaux sont morts 2 jours après, avec péritonite séro-fibrineuse et congestion des poumons et du foie; deux autres meurent 3 jours après l'inoculation, alors que la souche pathogène avec une dose moins forte $\left(0,2 \mathrm{~cm}^{3}\right)$ tue la souris en 8 à 10 heures.

L'hémoculture et l'ensemencement de la moelle des animaux morts régénèrent le bacille violet.

En résumé, il apparaît, après confrontation de ces divers résultats que:

$1^{0}$ L'infection est sous la dépendance de la virulence intrinsèque des souches, très variable quantitativement d'une souche à l'autre:

$2^{\circ}$ La réceptivité propre des espèces inoculées joue certainement un rôle important et permet de classer les espèces expérimentalement sensibles dans l'ordre de réceptivité croissante suivant: cobaye, lapin, souris, cette dernière espèce devant être considérée comme le réactif de choix.

$3^{\circ}$ L'absence de la bactérie cst constantc dans le sang au cours des infections chroniques, tandis que l'hémoculture est toujours positive dans les septicémies fulgurantes;

$4^{\circ}$ L'atteinte du poumon est régulière (pneumotropisme de la bacterie):

50 La sévérité est maxima par les voies intraveineuse et intranasale dans l'inoculation expérimentale.

\section{Pouvoir antigène.}

Dans le double but :

qualitatif de mettre en évidence des antigènes spécialement liés à la virulence de certaines souches de Chr. Violaceum;

quantitatif de rechercher le degré plus ou moins élevé de l'antigénicité des souches palhugènes vis-à-vis des souches saprophytes,

une série d'agglutinations 0 croisées a été réalisée entre les 4 souches étudiées.

La préparation des suspensions microbiennes a été ainsi effectuée. Des tubes de gélose nutritive inclinée glucosée à 0,1 p. 100 sont ensemencés avec une culture en bouillon glucosé à l p. 100 de la souche de Chromobacterium violaceum. Après 18 heures d'étuve à $37^{\circ}$, on récolte la culture en bouillon nutritif. Des boîtes de Roux contenant chacune $250 \mathrm{~cm}^{3}$ de gélose nutritive, sont ensemencées avec cette suspension à raison de $2 \mathrm{~cm}^{3}$ par boîte.
La récolte est effectuée en eau physiologique après 18 heures d'incubation à $37^{\circ}$. Ensuite une suspension' en eau physiologique contenant 20.109 germes (lavés 3 fois) par millilitre, est soumise à un chauffage à $100^{\circ}$ pendant 2 heures, pour détruire l'antigène $\mathrm{H}$ qui est commun à toutes les souches et ne semble jouer aucun rôle dans le pouvoir pathogène.

La préparation des sérums agglutinants a été réalisée de la manière suivante. Le producteur de serum choisi est le lapin. La préparation des sérums s'est faite soit à partir des suspensions microbiennes, soit à partir de l'extrait trichloracétique.

a) A partir des suspensions microbiennes. La suspension microbienne définie ci-dessus est diluée en eau physiologique stérile de façon à obtenir une densité égale à 500.10' germes par millilitre. Chaque lapin reçoit 5 injections intraveineuses à 5 jours d'intervalle: les trois premières injections ont été de $0,5,1,1,5 \mathrm{~cm}^{3}$; les deux dernières ont été faites avec $2 \mathrm{~cm}^{3}$. Les lapins ont été saignés une semaine après la dernière injection.

b) A partir de l'acide trichloracétique. - On injecte par voie veineuse au lapin, $10 \mathrm{~cm}^{3}$ d'extrait trichloracétique (cf supra) en 5 injections faites à 4 jours d'intervalle. Les lapins sont saignés une semaine après la dernière injection.

Les sérums ainsi préparés sont additionnés de $0,01 \%$ de merthiolate et conservés à $4^{0}$.

Les réactions d'agglutination admettent le protocole suivant $=\mathrm{X}$ gouttes de suspension microbienne sont mélangées avec $\mathrm{X}$ gouttes de sérum et la lecture est faite après 5 minutes de centrifugation à $2.000 \mathrm{t} / \mathrm{m}$. L'observation de la réaction est favorisée par l'emploi du miroir concave de Kahn.

Les résultats sont notés de la façon suivante:

+++ agglutinat dans un liquide clair,

++ agglutinat dans un liquide trouble,

+. agglutinat visible à l'œil nu,'

\pm agglutinat visible à la loupe.

Voici les résultats des réactions d'agglutination obtenus avec les sérums préparés avec les souches pathogènes 51.276 et 55.735 (tableau IV):

Le pouvoir agglutinant du serum des souches pathogènes est donc assez élevé vis-à-vis des souches homologues; il se maintient à la dilution de $1 / 320$ à $1 / 640$ et descend à $1 / 160$ avec les souches saprophytes hétérologues.

Les réactions d'agglutination des sérums venant des souches saprophytes sont les suivantes (tableau V): 
TABLEAU TT

\begin{tabular}{|c|c|c|c|c|c|c|c|c|c|c|c|c|}
\hline \multirow{2}{*}{\multicolumn{2}{|c|}{$\begin{array}{l}\text { SERTM PREPARE } \\
\text { AVDC SOUCEE }\end{array}$}} & \multirow{2}{*}{+} & \multirow{2}{*}{$\begin{array}{r}\text { CULTURE } \\
\text { SOUCHE }\end{array}$} & \multicolumn{9}{|c|}{ TITRE AGGLUTINATION } \\
\hline & & & & $1 / 20$ & $1 / 40$ & $1 / 80$ & $1 / 160$ & $1 / 320$ & $1 / 640$ & $1 / 1280$ & $1 / 2560$ & $1 / 5120$ \\
\hline Sérum & 51.276 & & 51.276 & $H$ & $++t$ & $H$ & $H$ & + & \pm & \pm & \pm & \pm \\
\hline$"$ & 51.276 & & 55.735 & +4 & +4 & + & H & + & \pm & 0 & 0 & 0 \\
\hline " & 51.276 & & SE 112 & H & ++ & + & + & \pm & 0 & 0 & 0 & 0 \\
\hline & 51.276 & & SE 115 & ++ & ++ & + & + & \pm & 0 & 0 & 0 & 0 \\
\hline & 55.735 & & 55.735 & $H$ & +++ & + & + & + & + & \pm & \pm & \pm \\
\hline & 55.735 & & 51.276 & + & ++ & + & $H$ & + & \pm & 0 & 0 & 0 \\
\hline & 55.735 & & SE 112 & $H$ & + & + & + & \pm & 0 & 0 & 0 & 0 \\
\hline & 55.735 & & SE 115 & $t+t$ & $H$ & $H$ & + & \pm & 0 & 0 & 0 & 0 \\
\hline
\end{tabular}

\section{TABLEAU $V$}

\begin{tabular}{|c|c|c|c|c|c|c|c|c|c|c|c|c|}
\hline \multirow{2}{*}{$\begin{array}{r}\text { SERUN } \\
\text { AVEC }\end{array}$} & \multirow{2}{*}{$\begin{array}{l}\text { PREPARE } \\
\text { SOUCHE }\end{array}$} & \multirow{2}{*}{+} & \multirow{2}{*}{$\begin{array}{l}\text { CULTURE } \\
\text { SOUCHE }\end{array}$} & \multicolumn{9}{|c|}{ TITRE AGGLUTINATION } \\
\hline & & & & $1 / 20$ & $1 / 40$ & $1 / 80$ & $1 / 160$ & $1 / 320$ & $1 / 640$ & $1 / 1280$ & $1 / 2560$ & $1 / 5120$ \\
\hline Sérum & SE 112 & & SE 112 & $H$ & ++ & H & + & \pm & 0 & 0 & 0 & 0 \\
\hline$"$ & SE 112 & & SE 115 & +4 & + & + & \pm & 0 & 0 & 0 & 0 & 0 \\
\hline$n$ & SE 112 & & 51.276 & + & + & + & + & \pm & 0 & 0 & 0 & 0 \\
\hline$"$ & $\mathrm{SE} 112$ & & 55.735 & ++ & + & + & + & \pm & 0 & 0 & 0 & 0 \\
\hline$"$ & SE 115 & & SE 115 & $H$ & $H$ & + & + & \pm & 0 & 0 & 0 & 0 \\
\hline & SE 115 & & SE 112 & ++ & $H$ & + & \pm & 0 & 0 & 0 & 0 & 0 \\
\hline " & SE 115 & & 51.276 & $H$ & $H$ & + & + & \pm & 0 & 0 & 0 & 0 \\
\hline$"$ & SE 115 & & 55.735 & $+H$ & + & + & + & \pm & 0 & 0 & 0 & 0 \\
\hline
\end{tabular}

L'agglutination est relativement faible avec les souches saprophytes: les souches homologues sont agglutinées au $1 / 160$, alors que les souches hétérologues ne sont agglutinées qu'au 1/80.

Dans l'ensemble, il existe donc peu de différence dans le pouvoir antigénique de ces 4 souches. Ces épreuves sérologiques croisées prouvent cependant:

10 Qualitativement, l'existence d'antigènes $O \mathrm{com}$ muns d'espèce et celle vraisemblable, d'antigènes $O$ de souche.

$2^{\circ}$ Quantitativement, l'existence d'antigènes plus «forts » chez les souches pathogènes. 


\section{SYMPTOIMES}

Chez l'animal (Ecuf et Buffle, Forc, Singe) on peut discerner 3 formes cliniquea de la chromobactériose:

Forme suraiguë, apoplectique, galopante, septicémique, qui tue en quelques heures et au plus 1 à 2 jours, sans livrer de signes plus caractéristiques que les septicémies pasteurelliques ou bactéridiennes.

L'animal présente une fièvre élevée, une prostration extrême. Les muqueuses sont cyanosées, la respiration dyspnéiquo, le facic̀s grippé, tous symptômes liés aux septicémies en général.

Forme aiguë, septicopyoémique qui développe en plusieurs jours et jusqu'à une à deux semaines des signes septicopyoémiques mineurs, d'une déconcertante diversité, intéressant tous les appareils ou se localisant au contraire à un ou deux d'entre eux. Il subsiste néanmoins un symptôme constant, illustrant le pneumotropisme de la bactérie responsable: l'atteinte constante du poumon, généralement sous la forme d'une bronchopneumonie sévère. - Les autres localisations entraînent des signes physiques et fonctionnels non pathognomoniques de l'infection.

Forme chronique, fréquente chez le Porc, avec dépression, anorexie, dyspnée. - Après un début fébrile, la maladie suit un cours /général favorable terminé par la guérison du sujet. Cependant une mortalité faible est constatée chez les porcs de gros format et il est difficile d'affirmer que les animaux guéris ne demeturent pas porteurs de germes. Les adénopathies superficielles sont rares, sauf chez le singe qui présente souvent un bubon axillaire et des plaies superficielles de la peau et des muqueuses.

Forme latente, infraclinique, qui se déduit logiquement de la présence ubiquitaire de Chr. violaceum dans les eaux tropicales et aussi de la sérologie positive montrée par certains sujets apparemment indemnes.

Chez l'homme. Ici, nous faisons un large emprunt au résumé clinique de Darrasse relevé sur un malade mort de chromobactériose. On a observé une température élevée $\left(40^{\circ} \mathrm{T}\right)$. Le malade était dyspnéique et très abattu avec un encombrement pulmonaire aux deux bases et des douleurs au niveau de l'hypocondre droit. On n'a pas remarqué de plaies ou autres lésions aux téguments. L'état du malade s'est aggravé d'heure en heure, la dyspnée s'accentuait et une cyanose intense s'installait avec un véritable « état de choc ":
L'hémoculture, pratiquée le jour de l'admission à l'hôpital révéla un bacille violet en culture pure.

\section{LÉSIONS}

Les lésions sont localisées surtout aux poumons, au foie, aux reins, au pancréas, Sur le porc, on note la fréquence de petits abcès dans la région parotidienne. Dans beaucoup de cas, les poumons sont adhérents à la cage thoracique en plusieurs points, sous l'effet d'une pleurésie symphysaire à développement relativement lent.

\section{Lésions macroscopiques.}

Poumons. - Les poumons congestionnés ont des abcès variant de $5 \mathrm{~mm}$ à $2 \mathrm{~cm}$ de diamètre. A l'incision, ces abcès laissent écouler un pus caséeux.

Foie. - Le foie a de nombreux abcès tuberculoïdes de $5 \mathrm{~mm}$ environ de diamètre, remplis de pus d'une fluidité supérieure à celle du pus des poumons. Les germes pullulent au niveau de la périphérie de ces abcès.

Rein. - Les reins sont congestionnés, mais on n'y constate ni abcès, ni hémorragies.

Pancréas. - Les abcès y sont rares, mais quand ils s'en présentent, ils sont de l'ordre de $4 \mathrm{~mm}$ à $1 \mathrm{~cm}$ et sont semblables à ceux du poumon.

On n'observe pas de lésions des ganglions mésentériques et inguinaux. Chez le porc, on trouve souvent un abcès de l'épiglotte contenant un pus. caséeux semblable à celui des autres lésions. Le tissu adjacent à cet organe renferme d'autres petits abcès clairsemés. Presque toujours enfin, les surrénales sont réactionnelles,

\section{$2^{\circ}$ Lésions microscopiques.}

Poumons. - Une infiltration des parois alvéolaires est généralement constatée, les « tubercules 》 sont constitués par une partie centrale contenant des lymphocytes ct des cellules nécrosés où les bacilles sont nombreux. Ces abcès nodulaires ressemblent macroscopiquement aux tubercules tuberculeux, mais ils s'en distinguent par l'absence de cellules géantes. On ne remarque pas de mésenchymatose, la nécrose se faisant sur place.

Rein. - On y rencontre des altérations épithéliales mineures et les cellules' nécroséés desquament. La plupart des tubes contournés conservent cependant des cellules à striation basale normale.

\section{DIAGNOSTIC}

\section{Épidémiologique.}

La stricte localisation de la chromobactériosé aux zones tropicales chaudes et humides représente 
un facteur épidémiologique de première importance. - Le caractère sporadique ou faiblement enzootique et la limitation de l'infection à quelques espèces animales seulement restreignent considérablement le champ d'un diagnostic épidémiologique différentiel et permettent souvent de séparer de cette infection les épizooties caractérisées, telle la pasteurellose. Le diagnostic est plus délicat si l'on considère la fièvre charbonneuse, maladie tellurique, à localisations géographiques, connues et soumise au rythme saisonnier.

\section{Clinique.}

La rapidite des formes aiguës et la diversité des évolutions chroniques refusent à la maladie de présenter des signes critères alors que la suspicion de chromobactériose coincide avec celle de melioildose, de fièvre charbonneuse ou de pasteurellose. Le diagnostic différentiel est encore rendu plus délicat par l'apparition d'adéno-phlegmons dans la mélioidose et d'une atteinte pulmonaire constante dans la pasteurellose. Seul le charbon bactéridien se distingue quelquefois par un syndrome hémorragique dont la violence extraordinaire ne se rencontre jamais dans la chromobactériose.

\section{Nécropsique.}

La présence d'abcès nodulaires tuberculoïdes sur les poumons, le foie, les reins et le pancréas constitue une lésion de suspicion mais non critère puisque les stigmates de cette septicopyoémie se Ietrouvent dans d'autres maladies tropicales, en particulier la mélioïdose.

\section{Expérimental.}

Bactérioscopie. - L'examen après coloration des lésions abcédées peut faire écarter certaines bactérioses (la fièvre charbonneuse surtout, à bacilles Gram +) mais demeure impuissante à distinguer les diverses infections à bactéries à Gram - (Pasteurelloses, Salmonelloses, Mélioïdose).

Cultures. - La facilité d'obtention en gélose ordinaire aérobie de colonies violettes révélatrices permet de ranger cette technique parmi les plus sûres à séparer les diverses septicémies bactériennes.

Inoculation. - La réussite de l'inoculation intranasale ou intraveineuse de pus infecté ou de culture pure à la souris blanche constitue un utile complément à l'isolement du germe mais n'apporte aucun élément diagnostique de grande valeur en raison de la sensibilité de ce Rongeur à tous les autres microbes tropicaux qu'il faut différencier de Chromobacterium. Ce test, associé aux autres tests in vitro (biochimiques en particulier) assure en revanche l'expérimentateur de posséder le responsable authentique d'une chromobactériose et non un germe « de sortie » avirulent. Cette recherche complémentaire de la virulence de la souche, exposée en détail au chapitre de la bactériologie, est aussi utile en ce domaine qu'en matière de Pasteurellose.

Sérodiagnostic. - Il n'est ni possible, ni nécessaire, puisque la maladie évolue d'une manière à ce point rapide que les anticorps ne peuvent apparaitre dans le sérum des animaux infectés et que, d'autre part, la caractérisation directe du germe est facile.

Cependant, le dépistage systématique des porteurs de germes pourrait bénéficier de cette technique, sinon dans un but prophylactique, du moins pour information et par ailleurs l'agglutination $O$ demeure un élément de distinction in vitro des souches pathogènes et saprophytes de Chr. violaceum.

Pronostic. - La maladie est médicalement grave et souvent mortelle dans ses formes évolutives rapides. Cependant la sensibilité du' germe aux antibiotiques majeurs (streptomycine, auréomycine) fait espérer des cures spectaculaires, dans la mesure où l'infection sera suspectée ou diagnostiquée à temps.

Economiquement négligeable en apparence, en raison de sa rareté, la chromobactériose infecte peut-être à l'état latent un nombre considérable d'animaux et en tue sans doute davantage que les les autopsies raisonnées le font supposer. La recherche systématique de la maladie pourrait en effet livrer des renseignements digne's d'intérêt et attirer l'attention sur le processus:

\section{ÉTIOLOGIE}

Matières virulentes. - Maladie hydrique, la chromobactériose reconnaît comme réservoir naturel les eaux de mares, marécages, cours d'eau lents des régions tropicales.

Dans la généralité des cas, c'est en effet l'eau de boisson qui a été incriminée dans l'étiologie de la maladie.

Les animaux malades et les cadavres n'en représentent pas moins un relai multiplicateur dangereux d'autant que ces bacteries font souvent retour au sol, au sous-sol, aux eaux terrestres et souterraines à la faveur d'un enfouissement mal compris.

\section{Résistance du germe.}

Le germe est fragile en général et cette constatation ne manque pas de surprendre chez un germe hydrotellurique. - La chaleur le tue à $55^{\circ}$ en 1 heure 
et le froid détruit à $0^{\circ}$ le germe alors que sa croissance est déjà entravée à +40 .

Cependant, le bacille montre une vitalité vigoureuse dans les conditions naturelles et sa conservation au laboratoire ne pose guère de problème.

Les agents chimiques sont actifs, en particulier le chlore: la javellisation de l'eau apparaît ainsi comme un excellent moyen de désinfection. Rappelons la sensibilité du germe à de nombreux antibiotiques.

\section{Réceptivité.}

L'espèce (maladie des bovins, des buffles, du porc, du singe, de l'Homme) et l'individu (maladie de « terrain 》) paraissent conditionner la réceptivité à la chromobactériose. Il est remarquable surtout de constater que les buffles et les porcs, de moeurs "semiaquatiques », paient le plus lourd tribut à l'infection. Mais subsiste le mystère de l'acquisition de virulence des souches saprophytes des eaux chez des animaux à terrain « préparé ».

Quant aux facteurs extrinsèques de la réceptivité, le mode d'élevage, l'alimentation, la saison, le climat ont un rôle important à jouer.

Modes de l'infection. - Non contagieuse au sens strict du terme, la chromobactériose paraît incapable de se transmettre d'un animal malade à un animal sain, mais tire son origine des eaux de boisson polluées.

On peut s'interroger sur le processus de l'éclosion d'une septicémie chromobactérienne à partir d'une infection latente infraclinique, ignorée. Une maladie initiale, une vaccination intempestive pourraient suffire à l'apparition d'une chromobactériose "de sortic » par opposition aux chromobactérioses essentielles classiques.

\section{Voies de pénétration.}

Dans la maladie spontanée, la voie digestive est la plus logique mais, en considérant le pneumotropisme de la bactérie, il n'est pas interdit d'imaginer une instillation trachéale (aspiration d'eau dans les voies respiratoires supérieures au moment de la déglutition) à l'origine d'une chromobactériose pulmonaire.

Les conditions expérimentales les plus favorables, nous l'avons $\mathrm{vu}$, consistent dans l'instillation intranasale ou l'inoculation intraveineuse de matériel infectieux à la souris blanche.

\section{PATHOGÉNIE}

La conjonction, nuancée à l'infini, de la réceptivité intrinsèque de l'hôte et de la virulence propre du germe offe les expressions anatomocliniques variées décrites ci-dessus:
- septicémie pure dans les cas rapides, àvec pullulation du germe par myriades dans le sang, les tissus, les organes;

- septicopyoémie retardée, qui évolue en deux phases distinctes: une septicémie initiale passagère, suivie d'une localisation viscérale avec abcès nodulaires.

- infection chronique localisée, où le germe ne se retrouve pas dans le sang, mais seulement dans les lésions suppuratives ou nécrotiques.

II en découle:

- la nécessité d'adresser au laburatoire des prélèvements judicieusement choisis en fonction de l'évolution du processus (sang et cis long dans les formes rapides, lésion localisée et pus dans les formes lentes);

- l'explication de l'échéc régulier des hémocultures dans les évolutions ralenties.

\section{PROPHYLAXIE}

En l'absence de prophylaxie médicale, seule des mesures sanitaires restent valables pour lutter contre une maladie difficile à combattre en raison de son caractère hydrotellurique.

, Les mesures rationnelles qu'il nous paraît logique de prendre ont trait:

$1^{\circ}$ Au dépistage systématique de la pollution des eaux par Chr. violaceum. Une « carte» sommaire de cette pollution pourrait être dressée et sa considération entraîner l'adoption des mesures suivantes.

$2^{\circ}$ A la défense des territoires non infectés en éloignant animaux et matériaux souillés.

$3^{\circ}$ A l'assainissement des zones polluées grâce : a. l'action sur le sol et les eaux (javellisation' des eaux, construction de chenaux d'irrigation dévasés, enfouissement des cadavres infectieux entre deux lits de chaux vive ou mieux destruction à l'équarrissage, de manière à éviter la surpollution des eaux et à réduire ou supprimer cette pollution.

à l'action sur les animaux de manière à éviter l'apparition de la maladie grâce à une protection hygiénique, la vaccination étant inconnue. Dans ce but, il sera nécessaire d'assurer aux animaux une eau de boisson saine ou désinfectée, et un parcage salubre en été, pendant la saison des pluies.

\section{TRAITEMENT}

Les renseignements manquent à ce chapitre et seul Sneath a signalé les heureux effets de l'auréomycine. - $\mathrm{Nul}$ doute que le spectre d'action très 
étendu des principaux antibiotiques majeurs (la pénicilline exceptée) et même des sulfamides ne réserve en ce domaine d'heureux lendemains.

\section{TRANSMISSION A L'HOMIME}

La contagiosité interhumaine ou de l'animal a l'homme n'a jamais été observée, ce qui doit faire refuser de ranger la chromobactériose au nombre des zoonoses. En particulier, on ne connaît aucun exemple de contagion dans l'entourage des malades, nii des exemples d'infection de laboratoire et de transferts de germes par des vêtements ou les objets usuels.

On ne peut évoquer actuellement qu'un mode de contagion indirecte par l'intermédaire de l'eau de boisson ou des aliments souillés. Cependant, outre la voie digestive, la peau pourrait peut-être servir aussi de porte d'entrée à la faveur de solutions de continuité même ténues.

La sensibilité particulière des sujets est liée à une défaillance brutale de l'organisme, ce qui explique que la chromobactériose soit surtout observée chez les surmenés, accidentés, choqués, intoxiqués ou individus souffrant d'une déficience physique grave, caractère que la maladie possède en commun avec la mélioïdose, la septicémie des morphinomanes.

\section{BIBLIOGRAPHIE}

1. AUDEBAUD (G.), GANZIN (M.), CECCALDI (J.) et MERVEILLE (P.). - Annales Inst. Pasteur, (1954), 87, 413 .

2. $\operatorname{BESSON}\left(A_{.}\right)$. - Technique microbiologique, 1 vol. Baillière éd. Paris, 1928 (p. 320).

3. BOIVIN (A.) et MEROSBEANU (L.). - Rev. Imm. (1935), 1, 555.

4. BREAUDAT. - Les Instituts Pasteur d'Indachine. Portail éd., Saïgon, 1922 (p. 196).

5. BRISOU (J.). - Microbiologie du milieu marin. Flammarion edit, Paris, 1955 (p. 41 ).
6. BROUDIN. - Les Instituts Pasteur d'Indochine. Portail édit., Saïgon, 1922 (p. 196).

7. BUTTIAUX (R.). - L'analyse bactériologique des eaux de consommation. ' 1 vol. Flammarion édit., Paris, 1951 (p. 48).

8. CHABBERT (Y.), -- Ann. Biol. Clin. (1951), 9, 544.

9. CORPE (W.-A.). - Jour. Bact. (1951), 62, 515.

10. DARRASSE (H.), MAZAUD (R.), GIUDICELLI (P.) et CAMAIN (R.). - Bull, SOC. Path. Exot. (1955), 48, 704.

$\because$. DUMAS $(\mathrm{H}$.$) . - Bactériologie médicale. Flam-$ marion édit., Paris, 1955 (p. 226).

12. ELTINGE (E.-T.). - Int. Bull, Bact, Nomencl. Tax. (1957), 7, 37 .

13. FLOCH (H.) et LAJUdIE (P. de). - Arch. Inst. Pasteur de la Guyane (1943). publ. no 63.

14. GILMAN (J.-P.). - Jour. Bact. (1953), 65, 1.

15. LEIFSON (E.). — Jour. Bact. (1956), 71, 393.

16. MAGROU (J.), PREVOT (A.-R.). - Ann. Inst. Pasteur (1948), 75, 99.

17. SATORY (A.), MEYER (J.), WOEDELE - C. $R$. Acad. Sc. $(1938), 208,960$.

18. SCHATTENBERG. - Jour. Bact. (1942), 44, 509.

9. SIPPEL (W.-L.), MEDINA (G.), ATWOOD (M.-B.). - Jour. Amer. Veter (1954), 124, 467.

2ר. SNEATH (P.H.A.), WHELAN (J.-P.), BRAGOVANSINGH (R.), et ELWARDS (P.). - The Lancet (1953), 47, 276.

21. STRONG (E.M.). - Science (1944), 100, 287.

22. TOBIE (W.-C.). - Jour, Bact. (1945), 49, 459.

23. TOPLEY et WILSON. - Principes of Bacteriology. Arnold édit. Londres, 1956 (p. 634).

24. WOLFF (A.). - Zentralb. Backt. (1911), 11, 30.

25. WOLley (P.-G.). - Bull. Bur. Phil. Is. (1934), $15,48$. 